\title{
A STUDY ON NASAL CARRIAGE OF MRSA AND ITS ANTIMICROBIAL SUSCEPTIBILITY PATTERN IN HEALTHY INDIVIDUALS AND HOSPITALISED PATIENTS.
}

Dr. M. Bharathi, Dr. I . Jyothi Padmaja.

1. Assistant Professor, Department of Microbiology, Andhra Medical College, Vishakapatnam.

2. Professor and HOD, Department of Microbiology, Andhra Medical College, Vishakapatnam.

\section{CORRESPONDING AUTHOR}

Dr. M. Bharathi, Assistant professor

Dept. of Microbiology,

Andhra Medical College,

Visakhapatnam, Andhra Pradesh, India.

E-mail: bharathikanthi65@gmail.com,

Ph: 9440615206.

ABSTRACT: BACKGROUND: Staphylococcus aureus is an important pathogen in hospital associated infections as well as in community acquired infections. About $20-40 \%$ of healthy persons carry Staphylococci in the nose. Most dreadly strains of S.aureus are Methicillin resistant S.aureus (MRSA) strains. Till recent times these MRSA strains were restricted to hospitals only as Health care associated MRSA (HA-MRSA). But now these strains have also emerged in community, called as Community associated MRSA (CA-MRSA).

AIM: 1. To study the nasal carriage rate of, Staphylococcus aureus in community and in hospital setting in adult males and it's Antimicrobial susceptibility pattern.

2. To compare the nasal carriage rate of HA-MRSA with that of CA-MRSA and their Antimicrobial susceptibility patterns. MATERIAL AND METHODS: Nasal swabs were collected from anterior nares of control group (100 healthy adult males with no risk factors for acquisition of HAMRSA) and study group (100 patients) from June 2011- August 2011. Samples were inoculated on Mannitol Salt Agar and incubated at $37^{\circ} \mathrm{c}$ for $24-48 \mathrm{hrs}$. Yellow colonies were considered as Staphylococcus aureus and after confirmation, they were subjected to antimicrobial susceptibility test by Modified Kirby-Bauer disc diffusion test. RESULTS: Nasal carriage rate of Staphylococcus aureus and MRSA was $14 \%$ \& 4\% in healthy individuals and $27 \%$ \& $11 \%$ in patients, with significant $p$ value. Community associated S.aureus and CA-MRSA were more susceptible to antimicrobials than Hospital associated S.aureus and HA-MRSA. All hospital strains and $85.78 \%$ community strains were resistant to penicillin. All strains were more susceptible to Amikacin and ciprofloxacin and least susceptible to co-trimoxazole. CONCLUSIONS: Nasal carriage rate of MRSA was higher in hospitalized patients than in general population. Resistance pattern was more in hospital strains.

KEY WORDS: Nasal carriage, CA-MRSA, HA-MRSA, Antimicrobial susceptibility pattern.

INTRODUCTION: Staphylococcus aureus is an important pathogen in hospital associated infections as well as in community acquired infections. About $20-40 \%$ of healthy persons carry Staphylococci in the nose. Most dreadly strains of S.aureus are Methicillin resistant S.aureus (MRSA) strains. Till recent times these MRSA strains were restricted to hospitals only as Health care associated MRSA (HA-MRSA). But now these strains have also emerged in community, called as Community associated MRSA (CA-MRSA). The known risk factors for CA-MRSA are 
antibiotic use in the past 3 months, hospitalization during past 12 months, diagnosis of skin and soft tissue infections (SSTIs) at admission [1], chronic illness, IV drug use, and close contact with health care personnel[2].

Although several studies were conducted on the nasal carriage of Saureus in school going children or in students and most of the Indian studies were on the prevalence of CAMRSA but not on HA-MRSA. Hence we made an attempt to study nasal carriage rate of community associated and health care associated S.aureus and MRSA in adult males.

AIM:

1. To study the nasal carriage rate of Staphylococcus aureus, in community and in hospital setting in adult males and its Antimicrobial susceptibility pattern.

2. To compare the nasal carriage rate of HA-MRSA with that of CA-MRSA and their Antimicrobial susceptibility patterns.

MATERIAL AND METHODS: Nasal swabs were collected from anterior nares from both control group and study group from June 2011- August 2011 after taking informed written consent. Institutional ethics committee approval was taken. Statistical analysis was done using ChiSquare method.

CONTROL GROUP: Comprised 100 healthy individuals in the age group of 20-40 yrs, who were not on antibiotics in the past 3 months, not hospitalized in the past $1 \mathrm{yr}$ and not having respiratory infections or skin and soft tissue infections at the time of study.

STUDY GROUP: Comprised post operative patients who were admitted to hospital for more than 1wk duration, without any ENT problem and were negative for nasal carriage of S.aureus at the time of admission.

A single sterile moistened swab was inserted into anterior nares and was rotated gently to collect nasal flora.

PROCESSING OF SAMPLE: Samples were inoculated on Mannitol salt agar (MSA), incubated at $37^{\circ} \mathrm{C}$ for $24-48 \mathrm{hrs}$. Processing was done by using standard methods [3]. Yellow colored colonies were considered as Staphylococcus and S.aureus was identified by positive, tube coagulase test and Voges Proskauer test and then subjected to antimicrobial susceptibility test by Modified Kirby-Bauer disc diffusion test using the following antibiotic discs - oxacillin $(1 \mu \mathrm{g})$, Penicillin $(10 \mathrm{u})$, Cotrimoxazole (Trimethoprim-Sulfamethoxazole: $1.25 / 23.75 \mu \mathrm{g})$, Vancomycin $(30 \mu \mathrm{g})$,

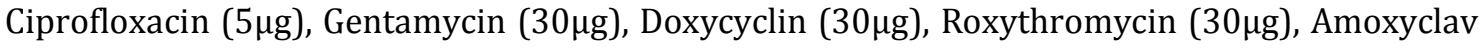

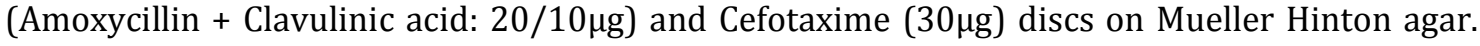
Next day susceptibility was determined by measuring diameter of zone of inhibition according to CLSI guide lines. Quality control was ensured by comparing the results with that of Staphylococcus aureus ATCC 25923 strain.

RESULTS: 14 persons (14\%) in control group and 27 patients (27\%) in study group were positive for nasal carriage of S.aureus. 4 of the 14 strains of S.aureus from control group (28.57\%) and 11 of the 27 strains from patients were MRSA (40.74\%) as shown in Table 1 and the values were statistically significant.

Journal of Evolution of Medical and Dental Sciences/Volume1/ Issue4/October-2012 Page 303 
All strains isolated from patients showed resistance to penicillin. $100 \%$ of community associated S.aureus (CA-SA) isolates and $96.29 \%$ of health care associated S.aureus (HA-SA) isolates were susceptible to Vancomycin. Least susceptibility was found to Cotrimoxazole. Antimicrobial susceptibility pattern of S.aureus and MRSA from both groups was shown in Table $2 \& 3$.

DISCUSSION: Many bacteria are transmitted from one person to another through hands. A person with S.aureus carriage in the anterior nares may rub his nose, pick up Staphylococci on the hands and spread the bacteria to other parts of the body or to other person, where infection results. The normal resident microbial flora is harmless, but if introduced into foreign locations, they may produce disease [4]. Nasal carriage of S.aureus acts as endogenous reservoir for clinical infections in the colonized persons, but also act as a source of cross colonization for others [5].

In the present study nasal carriage of S.aureus was $14 \%$ in control group and among them $28.57 \%$ were MRSA. In the test group it was $27 \%$ and among them $40.74 \%$ were MRSA. MRSA nasal carriage was $4 \%$ and $11 \%$ in control group \& test group respectively. In an unpublished study (2002) from the same author nasal carriage rate of S.aureus was $11.5 \%$ in burns patients at the time of admission and all strains were methicillin sensitive. It showed increase in the nasal carriage rate and development of resistance to methicillin over the years. Most of the Indian studies were on the prevalence of CA-MRSA but not on HA-MRSA. To the best of our Knowledge, our study was first of this kind in our area.

Nasal carriage of S.aureus in healthy adults was $14 \%$ in our study, $22.5 \%$ in the study of Goud R et al [6] and 46.1\% in Dev Jyothi Majumdar et al study [7] and 6.3\% in Pathak's study [5]. Rajendra Goud $\mathrm{N}$ et al [8] found it was $48.43 \%$ in the age group of $13-16$ yrs. The above studies were from India. Nasal carriage was $29 \%$ in 2 wks-21 yrs age group in a study by Nakamura [9], 29\% in students by Bischoff et al [10], 31.5\% in 20-64yrs age group in AG Mainous III et al study [11], 40.8\% in 18-27 yrs age group in Karina A Prates study [12] and $18 \%$ in healthy volunteers by Nicola Best [13]. The above findings suggested that there was a wide variation (14-52.3\%) in the nasal carriage of S.aureus among different age groups and in different geographical regions.

MRSA carriage rate in healthy adults also had wide range - $26.6 \%$ ( Dev Jyothi Majumdar), 9.9\% (Goud R), 0.84\% (AG Mainous III) , 0.3\%( Munckhof) [14], 2.4\% (Karina A Prates), $0.2 \%$ (Nicola Best) and 4\% (Present study) suggested higher rates in India than in other countries. CA-MRSA nasal carriage rate in the present study is lower than that from other areas in India.

Nasal carriage rate of HA-SA was $27 \%$ and HA-MRSA was $11 \%$ in the present study. No Indian studies were available in this regard. HA-MRSA carriage rate was 7.3\% in Al Hidron et al study [1] and $0.7 \%$ in Munckhof study [14] again shows the higher rate in India than in other countries.

In the present study $100 \%$ strains of CASA \& CA-MRSA, as in some studies $[2,8,12]$, $96.29 \%$ strains of HASA and $90.9 \%$ strains of HA-MRSA were susceptible to vancomycin, where as it was $56 \%$ in one study [15]. In an unpublished study (2002) from the same institute all MRSA strains were vancomycin sensitive, suggested development of vancomycin resistance in the sensitive strains, stresses the importance of adhering to hospital antibiotic policy. Indiscriminate use of antibiotics leads to development of resistance by selective pressure. Extensive use of penicillin for any infection especially in rural India results in the development 
of almost total resistance to penicillin. Similarly indiscriminate use of vancomycin may result in the development of vancomycin resistance in future. Hence the need of the hour is to take containment measures by strict implementation of antibiotic policy.

Least susceptibility was seen to co-trimoxazole, as in some studies $[5,15]$. Susceptibility of CA-S.aureus to amikacin, roxithromycin, ciprofloxacin, cefotaxime, doxycycline and amoxyclav was $78.57 \%, 57.14 \%, 64.28 \%, 78.57 \%, 64.28 \%$ and $57.14 \%$ respectively. Where as for HA-S.aureus it was $62.96 \%, 44.44 \%, 44.44 \%, 62.96 \%, 55.55 \%$ and $48.14 \%$ to the above drugs.

The antimicrobial susceptibility pattern of CA-MRSA varied widely among different studies. In the present study CA-MRSA strains were more resistant than CA-SA strains. HAMRSA strains showed still more resistance. The susceptibility rate of HA-MRSA to amikacin, roxithromycin, ciprofloxacin, cefotaxime, doxycycline and amoxyclav was 36.36\%, 36.36\%, $27.27 \%, 54.54 \%, 45.45 \%$ and $36.36 \%$ respectively. From the above findings it was clearly evident that hospital strains were more resistant to antimicrobials than community strains. Unless we take measurements, microbes will develop $100 \%$ resistance to these antibiotics also as in case of penicillin.

CONCLUSIONS: 1 . Nasal carriage rate of S.aureus and MRSA was higher in hospitalized patients than in healthy individuals.

2. Health care associated S.aureus and MRSA strains showed more resistance to antimicrobials than CA-SA and CA-MRSA.

Table 1 showing S.aureus and MRSA isolates from anterior nares of healthy individuals and patients

\begin{tabular}{|l|l|l|l|l|l|}
\hline Group & Total No. of cases & S.aureus* & $\%$ & MRSA $^{* *}$ & $\%$ \\
\hline Control & 100 & 14 & $14 \%$ & 4 & $4 \%$ \\
\hline Test & 100 & 27 & $27 \%$ & 11 & $11 \%$ \\
\hline
\end{tabular}

${ }^{*} \mathrm{p}$ value significant $(<0.05)$

** p value significant $(<0.05)$

Table 2 showing antimicrobial sensitivity pattern of S.aureus isolated from both groups in percentage

\begin{tabular}{|l|l|l|l|l|l|l|l|l|l|l|}
\hline Group & P & V & OX & CO & Ak & Ro & Cf & CTX & Do & AMC \\
\hline Control & 14.21 & 100 & 71.42 & 21.42 & 78.57 & 57.14 & 64.28 & 78.57 & 64.28 & 57.14 \\
\hline Study & 0 & 96.29 & 59.25 & 18.51 & 62.96 & 44.44 & 44.44 & 62.96 & 55.55 & 48.14 \\
\hline
\end{tabular}

Table 3 showing antimicrobial sensitivity pattern of CA-MRSA and HA-MRSA in percentage

\begin{tabular}{|l|l|l|l|l|l|l|l|l|}
\hline Group & V & CO & AK & RO & Cf & CTX & DO & AMC \\
\hline CA-MRSA & 100 & 25 & 75 & 75 & 50 & 75 & 75 & 75 \\
\hline HA-MRSA & 90.9 & 18.18 & 36.36 & 36.36 & 27.27 & 54.54 & 45.45 & 36.36 \\
\hline
\end{tabular}

AKNOWLEDGEMENTS: We are thankful to Dr. Krishnaveni, Professor, Dept. of Community Medicine for her guidance in statistical analysis. 


\section{REFERENCES:}

1. Alicia I. Hidron, Ekaterina V Kourbatova, Sue Halvosa, Bianca J. Terrell, Linda K. Mc Dougal, Fred C. Tenover, Henry M. Blumberg and Mark D. King " Risk factors for Colonization with Methicillin -Resistant Staphylococcus aureus (MRSA) in patients admitted to an Urban hospital: Emergence of Community- Associated MRSA Nasal carriage" Clin Infect Dis, 2005; 41 (2): 156-166.

2. Mandelia C, Shenoy S "Community- Associated - Methicillin Resistant Staphylococcus aureus in Skin and Soft tissue Infections" Journal of Clinical and diagnostic Research, 2010 August; (4):2673-7.

3. Mackie \&McCartney Practical Medical Microbiology.14thed, 2008: Publisher Churchill Livingstone. Chapter 11 :pp 247 \&253.

4. Jawetz,Melnick \& Adelbag's Medical Microbiology, 25 th Edition, publisher Mc Graw Hill company, 2010; Chapter 9 \&10 : pp $147 \& 160$.

5. Ashish Pathak A, Yogyata Marothi, Rama V Iyer, Binita Singh, Megha Sharma, Bo Eriksson, Ragini Macaden, Cecilia S Lundborg " Nasal carriage and Antimicrobial Susceptibility of Staphylococcus aureus in healthy preschool children in Ujjain, India" BMC Pediatrics 2010, 10: 100 doi: 10. 1186/1471-2431-10-100

6. Goud R, Gupta S, Neoqi U, Agarwal D. Naidu K, Chalannavar R, Subhaschandra "Community Prevalence of Methicillin and Vancomycin resistant Staphylococcus aureus in and around Bangalore, South India" Rev. Soc. Bras. Med. Trop. 2011, May-June; 44 (3): 309-12.

7. Dev Jyothi Majumdar, Ankur Barua, Barnali Paul. "Nasal carriage of methicillin resistant staphylococci in healthy population of east Sikkim." Indian Journal of Community Medicine 2009; 34(4): pp 364-365.

8. Rajendra Goud N, Deepali Agarval, Prasanth H Nadagoudar and Gaddad S M “ Antibiotic sensitivity pattern of Community- Associated Methicillin resistant Staphylococcus aureus (CA-MRSA) in High schools, Bangalore city, Karnataka, South India" International Medical Journal of Student's Research, October 2011; 1(1): 27-35.

9. Mari M Nakamura, Kasey L. Rohling, Michael Shashaty, Hongzhou LU, Yi-Wei Tang, and Kathryn M. Edwards "Prevalence of Methicillin- resistant Staphylococcus aureus Nasal carriage in the Community Paediatric Population" Pediatr Infect Dis J, 2002; 21 (10):917-21

10. Bischoff WE, Wallis ML, Tucker KB, Reboussin BA, Sheretz RJ "Staphylococcus aureus nasal carriage in a student community: Prevalence, clonal relationships and risk factors" Infect Control Hosp Epidemiol 2004 June; 25 (6): 485-91.

11. Arch G Mainous III, William J.Hueston, Charles J Everett and Vanessa A. Diaz "Nasal carriage of Staphylococcus aureus and Methicillin- resistant Staphylococcus aureus in the United States, 2001-2002" Annals of Family Medicine March 1, 2006; 4 (2): 132-7.

12. Karina Aparecida Prates, Ana Maria Torres, Lourdes Botelho Garcia, Sueli Fumia Yamada Ogatta, Celso Luiz Cardoso, Maria Cristina Bronharo Tognim "Nasal carriage of Methicillin- resistant Staphylococcus aureus in University Students" Brazilian Journal of Infectious Diseases, 2010; 14 (3) http:// dx. Doi. Org/10..1590/S1413-86702010000 300021

13. Nicola Best, John D Fraser, Paul B Rainey, Sally A Roberts, Mark G Thomas, Stephen R Ritchie "Nasal carriage of Staphylococcus aureus in healthy 
Aucklanders" Journal of The NewZealand Medical Association, 2011; vol 124, No 1332

14. Munckhof WJ, Nimmo GR, Schooneveldt JM, Schlebusch S, Stephens AJ, Williams G, Huygens F, Giffard P "Nasal carriage of Staphylococcus aureus including Community- Associated Methicillin- resistant strains in Queensland adults" Clin Microbiol Infect 2009; 15 (3): 296.

15. D H Tambekar, D V Dhanorkar, S R Gulhane, and M N Dudhane "Prevalence and Antimicrobial Susceptibility Pattern of Methicillin Resistant Staphylococcus aureus from Healthcare and community Associated sources" African J Infect. Dis 1 (1): $52-56$. 